\title{
Preimplantation genetic screening: a survey of in vitro fertilization clinics
}

\author{
Susannah Baruch, JD, David J. Kaufman, PhD, and Kathy L. Hudson, PhD
}

\begin{abstract}
Purpose: The purpose of this study was to determine which US in vitro fertilization clinics provide preimplantation genetic screening for aneuploidy in treating infertility, and to explore clinic directors' attitudes toward this technique. Methods: Online survey included 415 US assisted reproductive technology clinics. The survey had a valid response rate of $45 \%$ or 186 clinics. Results: Nearly $68 \%$ of US in vitro fertilization clinics responding to the survey provided preimplantation genetic screening in an effort to increase success rates of fertility treatment. More than half of these in vitro fertilization clinics (56\%) provided preimplantation genetic screening for advanced maternal age and the same percentage provided preimplantation genetic screening to treat repeated in vitro fertilization failure, whereas $66 \%$ provided preimplantation genetic screening to treat women with repeated miscarriage. Opinions of the effectiveness of preimplantation genetic screening for these indications varied widely, even among those providing it. Most directors (85\%) of clinics providing preimplantation genetic screening believed that more data are needed to determine whether and to whom it should be offered. Conclusions: Despite the lack of data supporting the use of preimplantation genetic screening for recurrent pregnancy loss, in vitro fertilization failure, and advanced maternal age, a majority of in vitro fertilization clinics in the United States offer preimplantation genetic screening for these purposes. There is significant support among clinic directors for more research into the effectiveness of preimplantation genetic screening and for professional guidelines in this area. Genet Med
\end{abstract} 2008:10(9):685-690.

Key Words: preimplantation genetic diagnosis, $P G D$, preimplantation genetic screening, $P G S$, in vitro fertilization, IVF, aneuploidy screening

New therapies in reproductive medicine may not be subject to full evaluation for safety and effectiveness before they are provided in a clinical setting. ${ }^{1}$ As in all areas of medicine, it is vital that data on uses, outcomes, and effectiveness of new assisted reproductive technologies be collected and critically evaluated.

Couples struggling with infertility now are being offered a relatively new technology in their quest to achieve a successful and healthy pregnancy. Preimplantation genetic diagnosis (PGD) is the genetic testing of embryos created through in vitro fertilization (IVF) to select embryos before transfer and implantation. PGD was first made available to prospective parents as an alternative to prenatal genetic diagnosis, typically to test embryos in cases where there was a known gene mutation

\footnotetext{
From the Genetics and Public Policy Center, Berman Institute of Bioethics, Johns Hopkins University, Washington DC.

Susannah Baruch, Genetics and Public Policy Center, Johns Hopkins University, 1717 Massachusetts Avenue NW, Suite 530, Washington DC 20036. E-mail: sbaruch2@jhu.edu.

Disclosure: The authors declare no conflict of interest.

Supplementary tables and appendices are available via the ArticlePlus feature at www.geneticsinmedicine.org.

Submitted for publication December 10, 2007.

Accepted for publication May 29, 2008.

DOI: 10.1097/GIM.0b013e31818180a1
}

in the family or where family history indicated the couple was at increased risk for having a child with a severe or deadly genetic disease. ${ }^{2,3}$

IVF providers now offer PGD to infertile couples who have no known genetic or inherited risks. ${ }^{4}$ This use of PGD is often called preimplantation genetic screening (PGS). ${ }^{4}$ The purpose of PGS is to evaluate embryos for abnormalities of chromosomal number, known as aneuploidy, which usually result in arrested or poor embryonic and fetal development and are believed to play a role in infertility, miscarriage, and IVF failure. ${ }^{3,5}$ It has been proposed that PGS may be used to improve pregnancy rates among infertile IVF patients not otherwise at risk for a genetic anomaly in their offspring. ${ }^{5,6}$ Data are mixed as to whether and for whom PGS is effective. . $^{3,78}$ Some studies support the use of PGS, particularly for patients experiencing recurrent miscarriages. ${ }^{9-11}$ Two recent studies have found that PGS did not improve implantation or pregnancy rates among women of advanced maternal age (AMA), and may in fact be detrimental. ${ }^{7,8,12}$ However, in general, few data have been collected or made available which systematically evaluate the practice of PGS in the United States-how often it is performed, for what indications, and with what outcomes. ${ }^{13,14}$ Despite conflicting studies and uncertainty about its safety and efficacy, demand for and availability of PGS are widespread. ${ }^{4,13}$ 
This study reports data on IVF clinic directors' practices and views with respect to the use of PGS - whether IVF clinic directors believed PGS improves the chance of a live birth (sometimes referred to as the "take-home baby rate") for infertile couples-and what formed the basis of their opinions. IVF clinics control whether and how infertile patients access PGS; thus clinic directors' experiences with, and views of, PGS are critical to understanding to what extent PGS is used, who is being given access to PGS, and how it may be used in the future.

\section{MATERIALS AND METHODS}

Between April 27, 2006 and May 31, 2006, the Genetics and Public Policy Center at Johns Hopkins University conducted an online survey of directors of all known US IVF clinics, or their designees. The study was approved by the Johns Hopkins Institutional Review Board. The sampling frame consisted of directors of 415 assisted reproductive technology (ART) clinics in the United States. The Society for Assisted Reproductive Technology (SART) provided contact information for each of the 393 SART member clinics. An additional 22 clinics were identified through publicly available information from the Centers for Disease Control and Prevention (CDC). ${ }^{15}$ Because all clinics performing ART are required under federal law ${ }^{16}$ to report data annually to the $\mathrm{CDC}$, and because most do so through SART, the sampling frame substantially represented all the clinics in the United States.

A draft survey was reviewed by five ART clinic directors and the research committee of SART. The final 87-question survey was administered online by Knowledge Networks through a secure website. Before the survey period, SART sent an e-mail to its member clinics introducing and endorsing the study. In this letter potential participants were informed that individually identifiable information collected during the survey would be kept confidential, and that data would be published only in the aggregate. Study invitations were mailed and e-mailed to each clinic, and reminder postcards and follow-up e-mails were sent to nonrespondents. Investigators recontacted two of the nonresponding clinics after the field period and both completed the survey on July 19, 2006.

To qualify for the survey and have their responses considered valid, clinic representatives had to confirm that their clinics currently offered IVF services, and that the respondent was a medical director, laboratory director, IVF director, or director's designee. Consent was electronic; by accessing the survey webpage and completing the survey, participants indicated their consent. Data were analyzed using a computer-based software package, SPSS version 14.0.1 (Chicago, IL). The survey questions used in this report are available at www.dnapolicy.org/resources/PGD_IVF_Survey.pdf.

\section{RESULTS}

A total of 190 directors or their designees responded to the survey. Of these, four failed to qualify because their clinics did not currently perform IVF, leaving 186 qualified respondents who completed the survey. The valid response rate was $45 \%$ and the qualification rate was $98 \%$. The median time respondents required to complete the survey was 21 minutes.

Although the observed $45 \%$ response rate is lower than typical survey goals of $60-70 \%$, methodologically sound surveys of healthcare professionals have experienced similarly low response rates. The clinics in the survey sample were highly comparable with data for 2005 on US IVF clinics reporting to the Centers for Disease Control with respect to both geographic location ( $\left.\chi^{2}=0.05,3 \mathrm{df}, P=0.997\right)$ and the distribution of IVF cycles completed in the past year $\left(\chi^{2}=5.32,3 \mathrm{df}, P=0.15\right)$ (Tables S1 and S2, available online).

Nearly three-quarters $(n=137)$ of the 186 qualified IVF clinics provided PGD for at least one indication. Among these 137 PGD clinics, 93\% $(n=127)$ provided PGS. These clinics reported performing a total of 2197 PGS cycles in 2005, which accounted for two-thirds of all PGD cycles in 2005. Of the 10 clinics that provided PGD but not PGS, three clinics said they offer PGS but no patient has requested it, and two clinics said they would offer aneuploidy screening in the future.

Extrapolating from our respondents to all IVF clinics, we estimated that approximately 5000 cycles of PGS were performed in US IVF clinics in 2005, representing an estimated 3-4\% of all reported IVF cycles (see Appendix, available online only). Among the 127 IVF clinics that provided PGS, only $15 \%(n=19)$ performed the genetic analysis of blastomeres in their own clinic laboratories. The remainder had the analysis performed in a laboratory outside their clinic. Only $5 \%(n=6)$ of the clinic laboratories that provided PGS said that they received blastomeres or polar bodies from other IVF clinics for analysis.

Directors who offer PGS were asked whether their clinics offer the screening for three distinct indications. More than half of all IVF clinics (56\%) offered PGS as a treatment for AMA. The same percentage of IVF clinics offered PGS to treat repeated IVF failure, and $66 \%$ of all IVF clinics offered PGS to treat women with repeated miscarriage (Table 1).

There was significant variation among PGS clinics with respect to the eligibility criteria for a patient to receive PGS. About half (53\%) of clinics providing PGS did not require a minimum number of embryos to proceed with genetic analysis. Among those with a stated minimum number of embryos, the average number of embryos required was 4.7 ; responses ranged from 3 to 7 .

More than two-thirds of clinics offering PGS for miscarriage and IVF failure had no minimum number of miscarriages or

Table 1

Indications for PGS among clinics that offer it

\begin{tabular}{lcc}
\hline Indication & $\begin{array}{c}\text { Percent of IVF } \\
\text { clinics offering PGS } \\
\text { for indication } \\
(n=186)\end{array}$ & $\begin{array}{c}\text { Percent of PGD clinics (clinics } \\
\text { offering PGD) offering } \\
\text { PGS for indication } \\
(n=137)\end{array}$ \\
\hline Advanced maternal age & 56 & 76 \\
Repeated IVF failure & 56 & 77 \\
Repeated miscarriage & 66 & 90 \\
\hline
\end{tabular}


Table 2

Criteria for PGS, by indication, among clinics offering PGS for the indication

\begin{tabular}{|c|c|c|c|}
\hline \multirow{3}{*}{$\begin{array}{l}\text { Indication } \\
\text { Repeated } \\
\text { miscarriage } \\
(n=123)\end{array}$} & \multirow{3}{*}{$\begin{array}{c}\text { Criteria } \\
\begin{array}{c}\text { Minimum number } \\
\text { of miscarriages }\end{array}\end{array}$} & \multicolumn{2}{|c|}{$\begin{array}{l}\text { Percent of clinics } \\
\text { providing PGS } \\
\text { for indication with } \\
\text { criteria }\end{array}$} \\
\hline & & No minimum & 68 \\
\hline & & One & 1 \\
\hline & & Two & 14 \\
\hline & & Three & 17 \\
\hline \multirow{4}{*}{$\begin{array}{l}\text { Repeated IVF } \\
\text { failure } \\
(n=105)\end{array}$} & Minimum number & No minimum & 74 \\
\hline & & Two & 10 \\
\hline & & Three & 15 \\
\hline & & Four & 1 \\
\hline \multirow{5}{*}{$\begin{array}{l}\text { Advanced } \\
\text { maternal age } \\
(n=104)\end{array}$} & AMA defined as & No definition & 6 \\
\hline & & $>34-35$ & 25 \\
\hline & & $>36-37$ & 17 \\
\hline & & $>38-39$ & 26 \\
\hline & & $>40$ & 26 \\
\hline
\end{tabular}

minimum number of IVF failures that must occur before a patient would be considered eligible for PGS. Among those with a stated minimum, there was no clear consensus about the criteria (Table 2). Similarly, although most clinics that offered PGS to treat AMA had a definition of AMA, the age defining AMA ranged from 34 to 43 (Table 2).

Clinic directors were asked several questions concerning their opinions about PGS. The overwhelming majority (85\%) of directors in clinics that provided PGS agreed that more data are needed to determine whether and to whom it should be offered. Most (89\%) directors of PGS clinics did not believe PGS should be offered to all or most IVF patients. However, $52 \%$ believed that PGS will be offered to all or most IVF patients in the next 10 to 15 years. Nearly half (47\%) of the directors who offered PGS agreed with the statement that "the push to offer PGD for aneuploidy screening is more about market pressure than medical evidence." Of those using PGS to treat miscarriage, 93\% felt it was a clinically valid tool for the indication. However, only $85 \%$ of those offering PGS for IVF failure and $79 \%$ of those offering PGS to treat AMA felt that the indications were clinically valid uses of PGS (Table 3). For all three indications, directors of the IVF clinics offering PGS for a given indication were significantly more likely than directors of clinics who did not offer PGS for that reason to agree that PGS was a clinically valid treatment for the indication (Table 3 ).

Respondents who provided any sort of PGD also were asked in an open-ended question to explain the basis for their opinions about PGS. Not all respondents named the basis of their opinion, but among those who did, the most commonly named sources of information (in order of decreasing frequency) were the scientific literature, the laboratory director's
Table 3

Beliefs about the clinical validity of different indications for PGS to treat infertility

\begin{tabular}{lccc}
\hline & \multicolumn{2}{c}{$\begin{array}{c}\text { Percent who believe PGS is clinically } \\
\text { valid tool to treat the indication }\end{array}$} & \\
\cline { 2 - 3 } & $\begin{array}{c}\text { Among IVF clinics } \\
\text { offering PGS for } \\
\text { the indication }\end{array}$ & $\begin{array}{c}\text { Among IVF clinics } \\
\text { not offering PGS } \\
\text { for the indication }\end{array}$ & P \\
\hline Indication & 93 & 71 & 0.0001 \\
\hline $\begin{array}{l}\text { Repeated miscarriage } \\
\text { Repeated IVF failure }\end{array}$ & 85 & 61 & 0.0002 \\
$\begin{array}{l}\text { Advanced maternal } \\
\text { age }\end{array}$ & 79 & 53 & 0.0003 \\
\hline
\end{tabular}

own experience with PGD for aneuploidy, presentations at meetings, and discussions with colleagues.

In the responses to the open-ended question about PGS, a large number of respondents expressed their opinions about the validity of PGD for aneuploidy. Clinic directors' views about the overall validity of PGS, and its validity for specific patient groups, were wide-ranging. Samples of these responses are found in Table 4. Some respondents raised concerns about the error rate, the possibility of misdiagnosis, and the possibility of harm to embryos from biopsy. Comments made by some directors that the evidence supports the use of PGS were contradicted by other directors who felt that evidence was clearly lacking.

\section{DISCUSSION}

For more than 25 years, ART and IVF have offered hope to patients struggling with infertility. ${ }^{17}$ It is estimated that more than one million children have been born in the United States after IVF, and IVF babies account for more than $1 \%$ of all births in the United States. ${ }^{18}$ PGS is a new tool available to IVF providers, and like IVF, its use seems to be increasing: it has become the most common reason that patients pursue PGD. ${ }^{4,13}$ PGS is available at two-thirds of IVF clinics in the United States, and expectations among IVF clinic directors are high that its use will increase in the future. ${ }^{13}$ However, clinic directors' opinions about the indications for, and effectiveness of PGS vary widely. Directors of clinics providing PGS express concerns that it has been adopted in clinical practice because of market pressures rather than medical evidence. Even among those providing PGS to patients for a given indication, there is little agreement about the criteria for administering PGS, and providers harbor questions about the validity of PGS for each indication. On the whole, directors strongly support the collection of more data on the safety and effectiveness of PGS and believe additional professional guidelines are needed for its use.

Studies examining the effectiveness of PGS have drawn a wide range of conclusions. Because a high rate of aneuploidy has been demonstrated among most groups of infertile patients, it initially seemed self-evident to many observers that PGS to screen out aneuploid embryos would improve fertility treatment outcomes. Thus far, evidence for the efficacy of PGS is strongest in studies of patients experiencing recurrent mis- 
Table 4

Sample of responses to the question "What is the basis of your views on aneuploidy?"

\begin{tabular}{|c|c|}
\hline & Positive comments \\
\hline \multirow[t]{2}{*}{$\begin{array}{l}\text { Evidence on outcomes } \\
\text { of PGS }\end{array}$} & $\begin{array}{l}\text { "It clearly improves the odds of transferring a normal embryo } \\
\text { (at least for the chromosomes we can test for)..." }\end{array}$ \\
\hline & $\begin{array}{l}\text { "Obvious benefits of improving delivery rates by reducing } \\
\text { miscarriage rates." }\end{array}$ \\
\hline
\end{tabular}

"Literature evaluations say to do it."

"I believe when validated it will allow us to improve pregnancy rates."

"The results are almost always valuable in some way: Patients with many embryos, the PGD results often help with selection (sometimes the best quality are not the 'normal' embryos); Patients with few embryos, the PGD results may not change the outcome, but usually shed some light on the patients['] prognosis."

"Benefits patients who do not become pregnant. By largely eliminating the period of pregnancy prior to miscarriage, patients may repeat IVF sooner after a failed cycle."

Appropriate indications

Accuracy

Effect of biopsy on embryo

\footnotetext{
"There is available evidence that it may diminish the incidence of miscarriage following IVF in female patients of advanced age."
}
"The delivery rates per cycle are NOT improved unless the indication is recurrent abortion."
"It may improve embryo implantation rates in patients with repetitive IVF failure."
"While there is clearly a risk of misdiagnosis due to mosaicism, the clinical error rate [is] very low and for many patients, the avoidance of a genetic termination is extremely important."
"I think PGD for aneuploidy is effective in identifying most embryos affected."
"The error rate is minimal vs. the increased chance of abnormal embryo being transferred."

"Does not harm embryo but may not be a true diagnostic tool."
Negative comments

"There is no data that shows that PGD for aneuploidy is helpful."

"In the literature, PGD patients are less likely to have a transfer. Data only looks good when comparisons are made using embryo transfer as a denominator."

“. . there is no data that I am aware of in the world [medical] literature that demonstrates improved pregnancy and delivery rates by use of PGD for aneuploidy screening."

"I feel it has limited use at the present time, but I also feel that it could become very important in the future."

"PGD is a waste of money and resources."

"I feel it is more of a market pressure offering, than clear scientific evidence."

"I strongly believe this is a market driven science and should only be used for single gene defects. Otherwise should be completed under IRB approved protocols."

"Lack of well designed studies demonstrating a difference in miscarriage or live birth. Need research with proper controls."

“... recurrent pregnancy loss has better prognosis without undergoing IVF."

"I feel PGD compromises the already fragile state of an AMA embryo."

"For advanced maternal age PGD is not effective usually due to too few embryos."

"Probably is only effective in patients at high risk for aneuploidy (e.g., recurrent pregnancy loss) and for patients with enough embryos that a selection can be made after testing."

"Too much mosaicism within embryos. Studies [show that it is] almost a coin toss when it comes to interpreting results."

"I worked at a large center where PGD was a huge thrust clinically and research-wise. We saw a large amount of misdiagnosis."

"Too high a false positive/negative rate. Analysis limited to only a few chromosomes at present."

"Poor embryo development after biopsy raises the question of the harm biopsy is doing to embryos."

"Pregnancy rates and implantation rates are adversely affected by the biopsy process." carriage. ${ }^{9,11}$ Several studies have shown that PGS reduces miscarriage rates among other IVF patients, including those of AMA. ${ }^{5,10}$ However, other studies contradict these findings. ${ }^{12,19}$

There is some research supporting the use of PGS for patients experiencing recurrent IVF failure ${ }^{3,20}$ and for IVF patients of AMA. ${ }^{3,9,20-22}$ However, several studies, including two that were randomized ${ }^{8,12}$ and one meta-analysis, ${ }^{7}$ found that PGS is not effective for women of AMA and may in fact be detrimental. ${ }^{8}$

In the most recent and widely reported study, Mastenbroek et al. ${ }^{8}$ randomized a large number of women and measured several different endpoints, from implantation to live birth. The study compared two groups of women aged 35-41. Controls went through normal IVF, whereas the experimental group had anueploidy screening of their embryos. Researchers compared the two groups' implantation rates after 7 weeks, pregnancy rates after 12 weeks, and live birth rates. Both pregnancy rates and birth rates were higher in the control group: the pregnancy rate for those who did not use PGS was 37\%, compared with $25 \%$ for those who did use PGS. Controls had significantly higher live-birth rate of 35\%, compared with $24 \%$ for the group given PGS. ${ }^{8}$ 
Some PGD experts have questioned whether studies such as that of Mastenbroek et al. ${ }^{8}$ that show less favorable results involved faulty PGS techniques. For example, some have suggested that the number of cells biopsied in the Staessen study, ${ }^{12,23}$ and that the high rates of undiagnosed embryos and embryo loss in the Mastenbroek study ${ }^{8}$ could have contributed to the less successful outcomes of PGS. ${ }^{24}$

The quality of much of the early data supporting the effectiveness of PGS has also been questioned. Some of the early studies of PGS involved very small numbers of patients. ${ }^{19,25,26}$ A Cochran review found fault with the first randomized studies of PGS for AMA for being inadequately randomized, and for using implantation rates rather than live birth rates as primary outcome measures. ${ }^{7}$ Among the nonrandomized studies, few have attempted to statistically control for simple confounders such as the age or past reproductive history of patients, or the number of embryos transferred, omissions that call their overall findings into question.

Wide variations in the study populations, indications and outcomes measured, and conclusions reached in the PGS literature, have also made it difficult to compare and interpret the findings of different studies. The strongest support for the use of PGS for AMA and repeated IVF failure has come from studies that rely on implantation rates as their main outcome. ${ }^{5,21,22}$ Few studies have reported data on ongoing pregnancy rates, and even fewer report data on live birth rates, the outcome that may be of strongest clinical relevance. ${ }^{7}$ Some studies simply fail to report any data or analysis of pregnancies or births. ${ }^{21,26}$ Other studies that have reported live birth rates as the primary outcome have not supported the use of PGS. In two studies of AMA, use of IVF with PGS was associated with higher rates of implantation or lower rates of miscarriage, but had no effect on pregnancy or live birth rate findings. ${ }^{5,22}$

There have been numerous calls for additional high-quality research into the effectiveness of PGS. 14,27,28 This call was supported by the vast majority of our survey respondents, who believed more data are needed to properly evaluate PGS and validate its use. As previously reported, the majority of clinic directors (62\%) said it would be a minimal burden to collect standardized data that would permit such research. ${ }^{13}$ Internationally, some valuable PGD data have been collected by the European Society for Human Reproduction and Embryology (ESHRE), but currently only $8 \%$ of US clinics providing PGS participate in ESHRE's PGD data collection. ${ }^{13}$ In the United States, the lack of comprehensive and standardized data collection has been a key challenge to the assessment of PGS. There have been public calls for a comprehensive PGD database in the United States. ${ }^{14,29}$ Efforts are underway to create such a database. ${ }^{14,29}$

Collecting standardized data will allow researchers to improve upon previous studies' methods, to analyze data consistently, and to determine exactly which patient groups-if any-are likely to benefit from PGS. As the gold standard for clinical research, randomized clinical trials of PGS across large patient populations will be necessary. There are a number of reasons these studies have not been done previously. First, such studies are expensive. Current US federal policies prohibit federal funding of research involving human embryos, making it unlikely that public funds would be used to support research on the effectiveness of PGS..$^{30}$ In countries without such policies and where the costs of IVF may be covered by national health services, some high-quality research has already been conducted. ${ }^{8,12}$ Second, unlike new drugs, medical devices, or biologics, which must receive approval by the Food and Drug Administration before coming to market, no federal agency requires techniques such as PGS to be reviewed before they are adopted in clinical practice: neither the biopsy procedure nor the genetic analysis receives federal oversight. It is not unusual for new medical techniques to develop in the course of clinical practice. Third, the demand for PGS may make it difficult for IVF clinics to delay offering it to fertility patients. There is considerable pressure on IVF clinics to compete in the market by adopting and offering new techniques such as PGS as soon as possible, sometimes before they are scientifically evaluated. As the number of prospective parents turning to IVF increases, the potential market for PGS will also increase, and the market pressure to offer and provide PGS will grow. The number of prospective mothers of AMA is on the rise- the US birthrate in women aged 35-39 increased 46\% between 1990 and 2005, from 31.7 births to 46.3 births per 1000 women. ${ }^{31,32}$ As demand for PGS grows, it is even more critical that it be thoroughly validated.

Experts have cautioned against broader applications of PGS until more definitive research on its efficacy is completed. 4,27,28 Standards of practice for PGS should also be considered. There have been increasing efforts to provide private, voluntary oversight of PGD by medical and scientific professional societies, including the American Society for Reproductive Medicine, the Preimplantation Genetic Diagnosis International Society, and ESHRE. ${ }^{33-38}$ Data previously published from this survey showed that a substantial majority of clinic directors believed that professional societies are best suited to create professional guidelines related to PGD (95\%) and that they should do so (85\%); however, most (79\%) opposed additional government oversight. ${ }^{13,36}$

Finally, it is important to note that there have been two significant developments since this survey was fielded. First, the largest study to date of the effectiveness of PGS, by Mastenbroek et al., ${ }^{8}$ published in the New England Journal of Medicine, found that PGS is not effective for infertility in women of AMA. Second, the American Society for Reproductive Medicine developed and published practice guidelines for the use of PGD and PGS and concluded that the available evidence does not support the use of PGS. ${ }^{38}$ These recent developments may have affected clinic practices and views, and the Genetics and Public Policy Center plans to study the impact on clinics by repeating this survey in the near future.

\section{CONCLUSION}

Patients desperate to increase their chances of having a healthy baby, and providers who refer patients to IVF clinics 


\section{Baruch et al.}

for help with fertility concerns, need evidence that PGS is effective and not merely an expensive add-on of questionable value. PGD for aneuploidy, or PGS, has emerged as a technology on the rise, but the best course for the future is continued robust research on the effectiveness of PGS and the development of additional evidence-based guidelines for its use.

\section{Acknowledgments}

The Genetics and Public Policy Center is supported at Johns Hopkins University by The Pew Charitable Trusts.

\section{References}

1. National Conference of State Legislatures. Genetics policy report: reproductive technologies. 2001.

2. Handyside AH, Kontogianni EH, Hardy K, Winston RM. Pregnancies from biopsied human preimplantation embryos sexed by Y-specific DNA amplification. $\mathrm{Na}$ ture 1990;344:768-770.

3. Verlinsky Y, Cohen J, Munne S, et al. Over a decade of experience with preimplantation genetic diagnosis: a multicenter report. Fertil Steril 2004;82:292-294.

4. Sermon KD, Michiels A, Harton G, et al. ESHRE PGD consortium data collection VI: cycles from January to December 2003 with pregnancy follow-up to October 2004. Hum Reprod 2007;22:323-336.

5. Munne S, Magli C, Cohen J, et al. Positive outcome after preimplantation diagnosis of aneuploidy in human embryos. Hum Reprod 1999;14:2191-2199.

6. Verlinsky Y, Kuliev A. Florescence in situ hybridization analysis of polar bodies and blastomeres. In: An Atlas of Preimplantation Genetic Diagnosis Parthenon; 2000:31-39.

7. Twisk M, Mastenbroek S, van Wely M, Heineman MJ, Van der Veen F, Repping S. Preimplantation genetic screening for abnormal number of chromosomes (aneuploidies) in in vitro fertilisation or intracytoplasmic sperm injection. Cochrane Database Syst Rev 2006;25:CD005291.

8. Mastenbroek S, Twisk M, Echten-Arends J, et al. In vitro fertilization with preimplantation genetic screening. N Engl J Med 2007;357:9-17.

9. Munne S, Chen S, Fischer J, et al. Preimplantation genetic diagnosis reduces pregnancy loss in women aged 35 years and older with a history of recurrent miscarriages. Fertil Steril 2005;84:331-335.

10. Munne S, Fischer J, Warner A, et al. Preimplantation genetic diagnosis significantly reduces pregnancy loss in infertile couples: a multicenter study. Fertil Steril 2006;85: $326-332$.

11. Verlinsky Y, Tur-Kaspa I, Cieslak J, et al. Preimplantation testing for chromosomal disorders improves reproductive outcome of poor-prognosis patients. Reprod Biomed Online 2005;11:219-225.

12. Staessen C, Platteau P, Van Assche E, et al. Comparison of blastocyst transfer with or without preimplantation genetic diagnosis for aneuploidy screening in couples with advanced maternal age: a prospective randomized controlled trial. Hum Reprod 2004;19:2849-2858.

13. Baruch S, Kaufman D, Hudson KL. Genetic testing of embryos: practices and perspectives of US. IVF Clinics. Fertil Steril 2008;89:1053-1058.

14. Baruch S, Adamson GD, Cohen J, et al. Genetic testing of embryos: a critical need for data. Reprod Biomed Online 2005;11:667-670.

15. Centers for Disease Control and Prevention. 2003 assisted reproductive technology (ART) report: appendix C. Available at: http://www.cdc.gov/ART/ART2003/appixc_ nonreport.htm. Accessed March 6, 2007.
16. Fertility clinic success rate and certification act of 1992. 42 USC Sec 263a-1.

17. Jones HW Jr. IVF: past and future. Reprod Biomed Online 2003;6:375-381.

18. Wright VC, Schieve LA, Reynolds MA, et al. Assisted reproductive technology surveillance-United States, 2002. MMWR Surveill Summ 2005;54:1-24.

19. Platteau P, Staessen C, Michiels A, et al. Preimplantation genetic diagnosis for aneuploidy screening in patients with unexplained recurrent miscarriages. Fertil Steril 2005;83:393-397.

20. Gianaroli L, Magli MC, Ferraretti AP, et al. The beneficial effects of preimplantation genetic diagnosis for aneuploidy support extensive clinical application. Reprod Biomed Online 2005;10:633-640.

21. Munne S, Sandalinas M, Escudero T, et al. Improved implantation after preimplantation genetic diagnosis of aneuploidy. Reprod Biomed Online 2003;7:91-97.

22. Gianaroli L, Magli MC, Ferraretti AP, Munne S. Preimplantation diagnosis for aneuploidies in patients undergoing in vitro fertilization with a poor prognosis: identification of the categories for which it should be proposed. Fertil Steril 1999;72:837844.

23. Cohen J, Munne S. Comment 2 on Staessen et al. (2004). Two-cell biopsy and PGD pregnancy outcome. Hum Reprod 2005;20:2363-2364.

24. Munne S, Gianaroli L, Tur-Kaspa I, et al. Substandard application screening may interfere of preimplantation genetic with its clinical success. Fertil Steril 2007;88: 781-784.

25. Stevens J, Wale P, Surrey ES, Schoolcraft W. Is aneuploidy screening for patient aged 35 or over beneficial? A prospective randomized trial. Fertil Steril 2004, 82(suppl 2):249.

26. Obasaju M, Kadam A, Biancardi T, et al. Pregnancies from single normal embryo transfer in women older than 40 years. Reprod Biomed Online 2001;2:98-101.

27. Collins JA. Preimplantation genetic screening in older mothers. N Engl J Med 2007; 357:61-63.

28. Shahine LK, Cedars MI. Preimplantation genetic diagnosis does not increase preg nancy rates in patients at risk for aneuploidy. Fertil Steril 2006;85:51-56.

29. The President's Council on Bioethics. Reproduction and responsibility: the regulation of new biotechnologies. Washington DC; 2004.

30. Balanced budged downpayment act I of 1996. Pub. L. No, 104-99, Sec 128, 110 Stat. 26, 34. 1996.

31. Hamilton BE, Martin JA, Ventura SJ. Births: preliminary data for 2005. Health e-stats. Released November 21, 2006. USDHHS CDC National Center for Health Statistics.

32. Martin JA, Hamilton BE, Sutton PD, et al. Births: final data for 2004. National vital statistics reports; vol 55 no 1. Hyattsville, MD: National Center for Health Statistics; 2006.

33. The Ethics Committee of the American Society of Reproductive Medicine. Sex se lection and preimplantation genetic diagnosis. Fertil Steril 1999;72:595-598.

34. Preimplantation Genetic Diagnosis. Fertil Steril 2004;82(suppl 1):S120-S122.

35. The Preimplantation Genetic Diagnosis International Society (PGDIS). Guidelines for good practice in PGD. Reprod Biomed Online 2004;9:430-434.

36. Simpson JL, Rebar RW, Carson SA. Professional self-regulation for preimplantation genetic diagnosis: experience of the American society for reproductive medicine and other professional societies. Fertil Steril 2006;85:1653-1660.

37. Thornhill AR, DeDie-Smulders CE, Geraedts JP, et al. ESHRE PGD consortium bes practice guidelines for clinical preimplantation genetic diagnosis (PGD) and preimplantation genetic screening (PGS)'. Hum Reprod 2005;20:35-48.

38. The Practice Committee of the Society for Assisted Reproductive Technology, the Practice Committee of the American Society for Reproductive Medicine. Preimplantation genetic testing: A Practice Committee opinion. Corrected Proof, 17 October 2007. Fertil Steril DOI: 10.1016/j.fertnstert. 2007.10.010. 2008. 